\title{
FuZZy Finite ELEMENT FREQUENCY RESPONSE ANALYSIS of STRUCTURES With UnCERTAin PARAMETERS
}

\author{
D. Vandepitte, D. Moens \\ Department of Mechanical Engineering, division PMA \\ K.U.Leuven, Celestijnenlaan 300B \\ 3001 Heverlee, Belgium \\ dirk.vandepitte@mech.kuleuven.ac.be
}

\begin{abstract}
$\underline{\text { ABSTRACT }}$
The finite element method is today a very common tool for both static and dynamic structural analysis. It is most often used in a deterministic procedure with precise values for all relevant parameters. In many cases however, geometrical or physical properties of the structure are not precisely known. Several sources of uncertainty can be identified. It is the aim of this work to incorporate all types of parameter uncertainties in a single analysis. A classical probabilistic approach is not suited for design validation purposes when the probability distributions have to be estimated. This is the case when there is limited or no statistical information on the uncertainties in an early design stage. The probabilistic result for those cases is unreliable and it may lead to false conclusions. For this reason, the concept of fuzzy numbers is used for the description of the uncertainties in the model. This concept forms a valuable alternative to numerically describe non-deterministic model properties. The analysis is performed by application of the concepts of the fuzzy finite element method. The core of this technique is the conservative approximation of the envelope FRF resulting from interval ranges for the uncertain inputs. The approach is first explained for undamped structures. The extension to damped structures is done for a proportional damping model. An example on a clamped plate shows practical results.
\end{abstract}

\section{INTRODUCTION}

The finite element (FE) method is a very popular tool for both static and dynamic structural analysis. The ability to predict the behaviour of a structure under static or dynamic loads is not only of great scientific value, it is also very useful from an economical point of view. A reliable FE analysis could reduce the need for prototype production and therefore significantly reduce the associated design validation cost. Unfortunately, it is very difficult to define a reliable FE model for realistic mechanical structures when a number of its physical properties are not known exactly. But even if the design is well defined, production inaccuracy and design tolerances introduce variability, resulting in a scatter of possible results for the analysis. Reliable validation can only be based on an analysis, which takes into account, all non-deterministic aspects of the design, including uncertainties and all identified sources of variability.

This paper focuses on the specific application of the finite element method for dynamic design validation. Requirements for the dynamic behaviour of a mechanical structure are commonly expressed in terms of its modal properties. Therefore, during the design phase of a mechanical structure much attention is paid to natural frequencies and mode shapes. However, these modal properties tend to vary strongly if small variations are applied on the design model. This means that the presence of uncertain parameters strongly complicates a meaningful dynamic design validation in an early design stage. It is clear that restricting the evaluation to one deterministic analysis of the modal properties in this case will not be sufficient. Probabilistic methods are available to calculate the effect of the model uncertainties on the analysis result. However, not every uncertainty in an early design stage can be exactly formulated in a probabilistic manner. Two

1

American Institute of Aeronautics and Astronautics 
problems have been identified:

- An actual variability, i.e. a property which will vary in the actual product is often not completely predictable, which leads to the introduction of assumptions concerning its probabilistic description.

- Other uncertainties in the model refer to properties which do not vary in the actual product, but are simply unknown or unspecified at an early design stage. The probabilistic model in this case represents personal opinion or preference of the designer rather than actual variability.

Due to these problems, it is more and more acknowledged that it is extremely difficult to perform a trustworthy probabilistic reliability study in an early design stage. It even has been shown that for some cases a probabilistic result can be incorrect and lead to false conclusions ${ }^{1}$.

The concept of fuzzy numbers provides in an alternative method to describe uncertainty. The concept allows for a linguistic description of the uncertainty in the model through fuzzy sets, and does not attach any probabilistic meaning to the numerical representation. The concept therefore explicitly incorporates subjectivity into the problem description. Using this concept for the numerical representation of uncertainties has initiated the development of a fuzzy finite element method (FFEM) ${ }^{2}$. The analysis result of this method however does not give an objective reliability measure as it is based on explicit subjective information. Therefore, it should be regarded more as a design optimisation and sensitivity analysis tool than an objective reliability prediction tool. Recently, a procedure to apply FFEM on the frequency response function analysis of undamped structures has been developed ${ }^{3}$. The objective of this paper is to demonstrate the technique and to extend its application to structures with proportional damping.

\section{The FuZZy Finite ELEMENT METHOD}

The main idea of the FFEM is to incorporate the fuzzy model parameters in the classic deterministic analysis procedure. This section of the paper first introduces the fuzzy arithmetic, which is applied to accomplish this. It further describes the consecutive steps of a typical FFEM analysis.
Fuzzy sets

The theory of fuzzy logic was introduced by ZADEH 4 in 1965, and has gained an increasing popularity during the last two decades. Its most important property is that it is capable of describing linguistic and therefore incomplete information in a non-probabilistic manner. This is achieved using the concept of fuzzy sets. A fuzzy set can be interpreted as an extension of a classical set. Where a classical set clearly distinguishes between members and non-members of the set, the fuzzy set introduces a degree of membership, represented by the membership function. This membership function describes the grade of membership to the fuzzy set for each element in the domain. The concept allows membership values different from zero and one. This enables the representation of a value that is only to a certain degree member of the set.

For a fuzzy set $\tilde{x}$, the membership function is defined as $\mu_{\tilde{x}}(x)$ for all $x$ that belong to the domain $X$ :

$$
\tilde{x}=\left\{\left(x, \mu_{\tilde{x}}(x)\right) \mid(x \in X)\left(\mu_{\tilde{x}}(x) \in[0,1]\right)\right\}
$$

If $\mu_{\tilde{x}}(x)=1, x$ is definitely a member of the subset $\tilde{x}$. If $\mu_{\tilde{x}}(x)=0, x$ is definitely not a member of the subset $\tilde{x}$. For every $x$ with $0<\mu_{\tilde{x}}(x)<1$, the membership is not certain. A class called normal fuzzy numbers is used here. For these fuzzy numbers, there is at least one point where the membership is equal to one, and the membership is strictly increasing and decreasing to the left respectively the right of this point. The most frequently applied shapes for the membership functions are the triangular and Gaussian shape.

\section{Fuzzy arithmetic}

Historically, a number of concepts have been introduced to perform numerical operations on fuzzy variables. The most widely known concept consists of a sequence of max-min operations on a numerical representation of the membership functions of the fuzzy parameters. Since the size of the representation of the fuzzy result grows exponentially with every operation, this concept is rarely applied in large numerical computations. The $\alpha$-sublevel technique is a valuable alternative. It subdivides the membership range into a number of $\alpha$-levels. The intersection with the membership function of the input uncertainties at each level results in an interval. With these input intervals of the $\alpha$-sublevel, an interval analysis corresponding to 

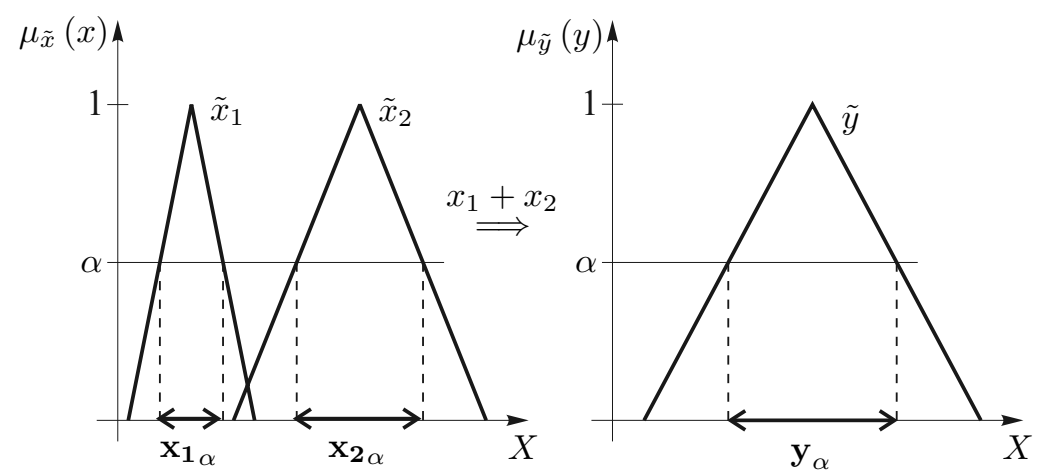

Figure 1: Calculation of the result of the function $f\left(x_{1}, x_{2}\right)=x_{1}+x_{2}$ by discretisation of the range of the membership function using the $\alpha$-cut strategy.

the deterministic analysis is performed. This results in an interval for the analysis result at the considered $\alpha$ level. Finally, the fuzzy solution is assembled from the resulting intervals at each sublevel, repeating this procedure for a number of $\alpha$-sublevels. Figure 1 clarifies this procedure for a function of two triangular fuzzy variables.

The core problem of this procedure is the calculation of the result of the function in interval arithmetic. An interval is denoted by a boldface variable $\mathrm{x}=[\underline{x}, \bar{x}]$. For a function of $n$ fuzzy variables $f\left(\tilde{x}_{1}, \tilde{x}_{2}, \ldots, \tilde{x}_{n}\right)$, the result is calculated at each $\alpha$ sublevel from the solution set defined as

$$
\begin{array}{r}
\mathbf{y}_{\alpha}=\left\{y \mid\left(\exists x_{i} \in \mathbf{x}_{\mathbf{i}_{\alpha}}, \forall i=1, \ldots n\right) \ldots\right. \\
\left.\left(y=f\left(x_{1}, x_{2}, \ldots x_{n}\right)\right)\right\}
\end{array}
$$

with $\mathbf{x}_{\mathbf{i} \alpha}$ describing the interval of the fuzzy variable $\tilde{x_{i}}$ at level $\alpha$.

This technique guarantees a constant size of the data set during computation. Another advantage is that the calculation of a fuzzy function can be optimized to a trade-off between computational effort and correctness by selecting the number of $\alpha$-sublevels.

General concept of the Fuzzy Finite Element analysis

The fuzzy finite element method as introduced by Chen ${ }^{5}$ consists of the application of the fuzzy arithmetical procedure through the $\alpha$-sublevel strategy on the numerical procedure of the deterministic FEM analysis. From the previous section it is clear that the numerical core of the method consists of the solution of the interval problem corresponding to the FEM analysis. A number of concepts have been developed for this purpose. The most common is the interval arithmetical approach. It consists of the translation of the total deterministic algorithm, which performs the FEM analysis to interval arithmetic, using the interval counterparts of all basic operations. As such, the procedure follows completely the outline of the deterministic counterpart. It generally consists of two steps:

1. the translation of the input intervals to an interval system description in the form of interval system matrices

2. the approximation of the solution of the analysis expressed as an interval problem using the interval system matrices

The first step requires the interval system assembly, which results from translating the deterministic assembly procedure to interval analysis. It is clear that this step depends strongly on the FEM application (heat conduction problem, structural analysis, ...) and the considered element types. Generally, this step results in a number of interval system matrices denoted by $\left[\mathbf{A}_{\mathbf{1}}\right],\left[\mathbf{A}_{\mathbf{2}}\right], \ldots\left[\mathbf{A}_{\mathbf{m}}\right]$ with $m$ depending on the problem.

The second step consists of the solution of the interval problem:

$$
\begin{array}{r}
\mathbf{y}=\left\{y \mid\left(\exists\left[A_{i}\right] \in\left[\mathbf{A}_{\mathbf{i}}\right], i=1, \ldots m\right) \ldots\right. \\
\left.\left(y=f_{A}\left(\left[A_{1}\right], \ldots\left[A_{m}\right]\right)\right)\right\}
\end{array}
$$


with $f_{A}$ the function representing the calculation of the deterministic analysis result based on the system matrices. The interval system matrix assembly is generally an easy procedure. Therefore, the applicability of the interval arithmetical strategy depends mainly on the availability of a calculation procedure for the interval problem appearing in the second step of the analysis.

\section{FFEM FOR FREQUENCY RESPONSE ANALYSIS}

Interval arithmetical FFEM procedure

This section considers the analysis at a specified $\alpha$ level. For the sake of clarity, the $\alpha$ index is omitted from the equations. Consider a structure with $n$ uncertain input parameters $x_{i}$ contained in an interval $\mathbf{x}_{\mathbf{i}}$. In the case of undamped structural dynamic response analysis, the procedure described in the previous section consists of

1. the assembly of the interval system stiffness and mass matrix:

$$
\begin{array}{r}
{[\mathbf{K}]=\left\{K(\{x\}) \mid\left(\exists x_{i} \in \mathbf{x}_{\mathbf{i}}, \forall i\right)\right\}} \\
{[\mathbf{M}]=\left\{M(\{x\}) \mid\left(\exists x_{i} \in \mathbf{x}_{\mathbf{i}}, \forall i\right)\right\}}
\end{array}
$$

with $K(\{x\})$ and $M(\{x\})$ representing explicitly the dependency of the system matrices on the uncertain input parameters represented by the vector $\{x\}$

2. the calculation of the solution set

$$
\begin{aligned}
\{\mathbf{y}\}= & \{\{y\} \mid(\exists[K] \in[\mathbf{K}],[M] \in[\mathbf{M}]) \\
& \left.\ldots\left(\left([K]-\omega^{2}[M]\right)\{y\}=\{f\}\right)\right\}
\end{aligned}
$$

with $\{f\}$ the load force vector and $\{\mathbf{y}\}$ the interval response vector

There are three objections to this methodology.

- First, the assembly of the interval system matrices in the first step results from the translation of the deterministic assembly procedure to interval analysis. This generally introduces conservatism, since dependency between different elements of the model through for instance a common material parameter are lost in the assembly. Furthermore, the system interval matrix does not model the dependencies between the elements of the matrix itself, and looses as such important information on internal matrix correlations. The resulting interval matrices therefore implicitly incorporate artificial matrices in the analysis, which are not feasible using the physical FEM model. For realistic industrial sized FEM models, this conservatism could render the rest of the analysis little useful.

- Secondly, it has been shown that the calculation of the exact solution set as expressed in equation (6) is not possible. Only an approximate solution is possible within an acceptable timeframe. Most approximation techniques however further increase the conservatism, and as such intensify the first objection.

- Finally, it is clear that if the analysis is considered over a large frequency domain, the solution of equation (6) is needed at a large number of discretised frequencies. This increases the computational cost.

\section{Modal superposition FFEM procedure}

From the objections in the previous section it is clear that the FFEM procedure based exclusively on interval arithmetic is not a good approach for FRF analysis. Therefore, a methodology based on the modal superposition principle is introduced. The deterministic concept states that, considering the first $n_{\text {modes }}$ modes, the frequency response function between node $j$ and $k$ equals

$$
F R F_{j k}=\sum_{i=1}^{n_{\text {modes }}} \frac{\phi_{i_{k}} \phi_{i_{j}}}{\phi_{i}^{T}[K] \phi_{i}-\omega^{2} \phi_{i}^{T}[M] \phi_{i}}
$$

with $\phi_{i}$ the $i^{t h}$ eigenvector of the system. Simplification of (7) yields

$$
F R F_{j k}=\sum_{i=1}^{n_{\text {modes }}} \frac{1}{\hat{k}_{i}-\omega^{2} \hat{m}_{i}}
$$


with $\hat{k}_{i}$ and $\hat{m}_{i}$ the normalised modal parameters

$$
\begin{aligned}
\hat{k}_{i} & =\frac{\phi_{i}^{T}[K] \phi_{i}}{\phi_{i_{j}} \phi_{i_{k}}} \\
\hat{m}_{i} & =\frac{\phi_{i}^{T}[M] \phi_{i}}{\phi_{i_{j}} \phi_{i_{k}}} .
\end{aligned}
$$

The translation of this algorithm to interval arithmetic results in three basic steps:

1. the direct transformation of the interval input parameters to the modal space for each considered mode:

$$
\begin{array}{r}
\hat{\mathbf{k}}_{\mathbf{i}}=\left\{\frac{\phi_{i}^{T}[K] \phi_{i}}{\phi_{i_{j}} \phi_{i_{k}}} \mid(\exists\{x\} \in\{\mathbf{x}\}) \ldots\right. \\
\left.\left(K(\{x\}) \phi_{i}=\lambda_{i} M(\{x\}) \phi_{i}\right)\right\} \\
\hat{\mathbf{m}}_{\mathbf{i}}=\left\{\frac{\phi_{i}^{T}[M] \phi_{i}}{\phi_{i_{j}} \phi_{i_{k}}} \mid(\exists\{x\} \in\{\mathbf{x}\}) \ldots\right. \\
\left.\left(K(\{x\}) \phi_{i}=\lambda_{i} M(\{x\}) \phi_{i}\right)\right\}
\end{array}
$$

in which $\hat{\mathbf{k}}_{\mathbf{i}}$ and $\hat{\mathbf{m}}_{\mathbf{i}}$ represent the possible values the modal stiffness and mass of the $i^{t h}$ mode can adopt taking into account all values within the specified ranges for the input parameters

2. the calculation of an approximation of the interval FRF contribution of each mode using the interval modal parameters:

$$
\begin{array}{r}
\mathbf{F R F}_{\mathbf{j k}}^{\mathbf{i}}=\left\{\frac{1}{\hat{k}_{i}-\omega^{2} \hat{m}_{i}} \mid\left(\exists \hat{k}_{i} \in \hat{\mathbf{k}}_{\mathbf{i}}\right) \ldots\right. \\
\left.\left(\exists \hat{m}_{i} \in \hat{\mathbf{m}}_{\mathbf{i}}\right)\right\}
\end{array}
$$

3. the summation of the contribution of each mode to obtain the total interval FRF:

$$
\mathbf{F R F}_{\mathbf{j k}}=\sum_{i=1}^{n_{\text {modes }}} \mathbf{F R F}_{\mathbf{j k}}^{\mathbf{i}}
$$

The first step is performed using an optimisation procedure in which the modal parameters are the goal function and the input parameters $x_{i}$ are the design variables with constraints defined by the corresponding interval $\mathbf{x}_{\mathbf{i}}$. The second step proves to be an analytical procedure. The calculation based on independent modal parameters however introduces conservatism in this step, because these parameters are actually coupled through the global system. It has been shown that the approximation can be greatly improved by using information on the eigenvalue ranges, which can be obtained using an eigenvalue optimisation step ${ }^{6}$. The final step is the easiest as it consists only of adding together the boundaries of the individual interval ranges over the considered frequency domain.

The advantages of this procedure are:

- the conservatism introduced by the interval system matrix assembly phase has disappeared using the optimisation directly on the modal parameters

- the computational cost is controllable through a selection of the modes which contribute to the total FRF in the frequency domain of interest

- there is no need for a discretisation of the frequency domain

\section{Proportionally damped structures}

For proportionally damped structures, the damping matrix follows directly from the global stiffness and mass matrix using:

$$
[C]=\alpha_{K}[K]+\alpha_{M}[M]
$$

with $\alpha_{K}$ and $\alpha_{M}$ proportional constants to be defined for each analysis. This is one of the most common models of damping. The assumption of proportional damping is purely made for mathematical convenience as it simplifies the solution process. The proportional damping model has been proved to be reliable for structures with damping below $10 \%$ of critical ${ }^{7}$.

Using the proportional damping model, the deterministic total FRF yields:

$$
F R F_{j k}=\sum_{i=1}^{n_{\text {modes }}} \frac{1}{\hat{k}_{i}+\jmath \omega\left(\alpha_{K} \hat{k}_{i}+\alpha_{M} \hat{m}_{i}\right)-\omega^{2} \hat{m}_{i}}
$$

Again, the modal stiffness and mass are the only parameters that vary with the uncertain input parameters. Therefore, step 1 of the undamped procedure remains unchanged. The second step of the algorithm now concentrates on the real and imaginary part of the modal FRF contributions. Both can be written as an explicit function of the modal parameters and the proportional 
damping constants:

$$
\begin{aligned}
& \Re\left(F R F_{j k}^{i}\right)=\frac{\hat{k}_{i}-\omega^{2} \hat{m}_{i}}{\left(\hat{k}_{i}-\omega^{2} \hat{m}_{i}\right)^{2}+\omega^{2}\left(\alpha_{K} \hat{k}_{i}+\alpha_{M} \hat{m}_{i}\right)^{2}} \\
& \Im\left(F R F_{j k}^{i}\right)=\frac{-\omega\left(\alpha_{K} \hat{k}_{i}+\alpha_{M} \hat{m}_{i}\right)}{\left(\hat{k}_{i}-\omega^{2} \hat{m}_{i}\right)^{2}+\omega^{2}\left(\alpha_{K} \hat{k}_{i}+\alpha_{M} \hat{m}_{i}\right)^{2}}
\end{aligned}
$$

The procedure calculates the envelope of both the real and imaginary part separately. These can be derived analytically based on the interval modal parameters ${ }^{6}$. This results in the real and imaginary modal envelope FRF's:

$$
\begin{array}{r}
\Re\left(\mathbf{F R F}_{\mathbf{j k}}^{\mathbf{i}}\right)=\left\{\Re\left(F R F_{j k}^{i}\right) \mid\left(\exists \hat{k}_{i} \in \hat{\mathbf{k}}_{\mathbf{i}}\right) \ldots\right. \\
\left.\left(\exists \hat{m}_{i} \in \hat{\mathbf{m}}_{\mathbf{i}}\right)\right\} \\
\Im\left(\mathbf{F R F}_{\mathbf{j k}}^{\mathbf{i}}\right)=\left\{\Im\left(F R F_{j k}^{i}\right) \mid\left(\exists \hat{k}_{i} \in \hat{\mathbf{k}}_{\mathbf{i}}\right) \ldots\right. \\
\left.\left(\exists \hat{m}_{i} \in \hat{\mathbf{m}}_{\mathbf{i}}\right)\right\}
\end{array}
$$

The final step depends on the intended analysis result. The real and imaginary part of the total FRF result directly from the summation of the corresponding individual modal FRF contributions:

$$
\begin{aligned}
& \Re\left(\mathbf{F R F}_{\mathbf{j k}}\right)=\sum_{i=1}^{n_{\text {modes }}} \Re\left(\mathbf{F R F}_{\mathbf{j k}}^{\mathbf{i}}\right) \\
& \Im\left(\mathbf{F R F}_{\mathbf{j k}}\right)=\sum_{i=1}^{n_{\text {modes }}} \Im\left(\mathbf{F R F}_{\mathbf{j k}}^{\mathbf{i}}\right)
\end{aligned}
$$

From these, the amplitude and phase interval of the total FRF are derived using:

$$
\begin{gathered}
\left|\mathbf{F R F}_{\mathbf{j k}}\right|=\sqrt{\Re\left(\mathbf{F R F}_{\mathbf{j k}}\right)^{2}+\Im\left(\mathbf{F R F}_{\mathbf{j k}}\right)^{2}} \\
\varphi\left(\mathbf{F R F}_{\mathbf{j k}}\right)=\varphi\left(\frac{\Im\left(\mathbf{F R F}_{\mathbf{j k}}\right)}{\Re\left(\mathbf{F R F}_{\mathbf{j k}}\right)}\right)
\end{gathered}
$$

This final amplitude and phase calculation forms a new source of conservatism since it combines the real and imaginary contributions as if they were independent, while they are coupled through the global system.

\section{NUMERICAL EXAMPLE}

The fuzzy FE analysis with modal superposition is run on a square steel $5 \mathrm{~mm}$ plate $1 \times 1 \mathrm{~m}^{2}$ as indicated in figure 2. The plate is clamped at one edge with a finite stiffness vertical translational boundary condition $[0.4,40] M N / m$. The other degrees of freedom at this edge are fixed. Proportional damping of about $1 \%$ is

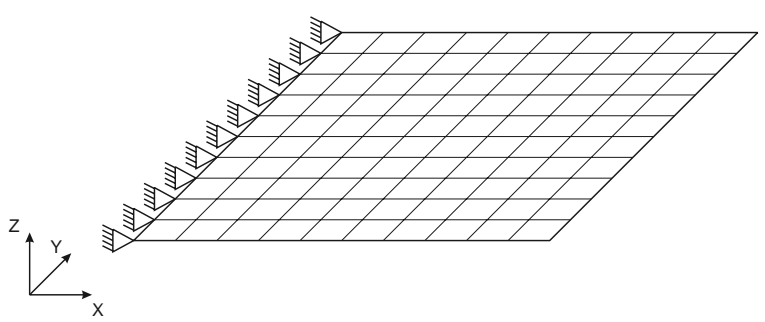

Figure 2: Plate model with finite fuzzy stiffness on rotational dof at the clamping

assumed. The excitation $F$ is applied at one corner, and vertical displacement is calculated at the other free corner.

The envelope FRF is calculated and compared to a set of Monte Carlo samples, with a logarithmic distribution in the boundary stiffness factor. Figure 3 shows the boundaries of the interval range, and the MC samples. The boundaries closely match the MC samples, except at some frequencies where the interval envelope seems to be too wide.

The same interval result is then compared to a set of Monte Carlo samples, with a linear distribution in the boundary stiffness factor. Figure 4 shows the boundaries of the interval range, and the MC samples.

The same problem is further analysed with another uncertain parameter, the finite stiffness of the rotational degree of freedom at the constraint : $[0.1,1] \mathrm{kNm} / \mathrm{rad}$. A triangular membership function is assumed. The other degrees of freedom are now fixed. Figure 5 gives the resulting fuzzy FRF with the degree of membership. This fuzzy FRF result gives a clear indication of the variation of the FRF over the considered frequency domain. It illustrates the sensitivity of the response to the rotational stiffness at the clamping.

\section{CONCLUSION}

This paper gives an overview of the application of the fuzzy finite element method for frequency response function analysis of uncertain damped structures. Three important difficulties arise in the application of the classical interval arithmetical approach. 

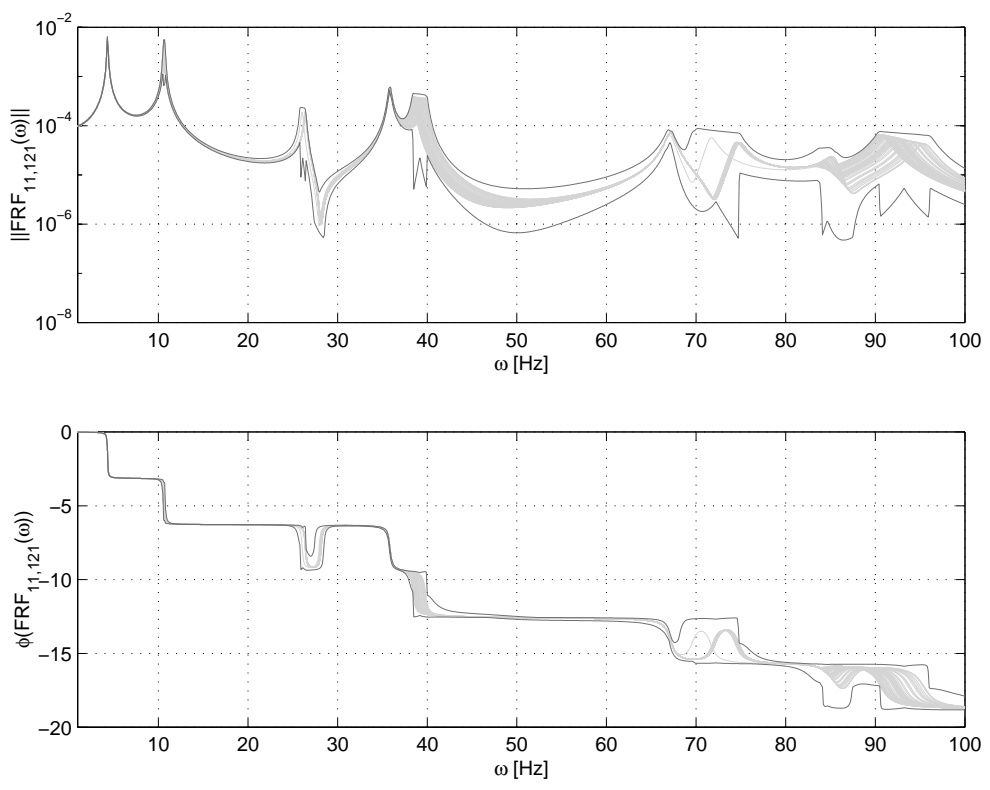

Figure 3: Envelope FRF for clamped plate with uncertain vertical boundary stiffness (MC with logarithmic distribution)

Therefore, a methodology based on the deterministic modal superposition principle has been developed. This concept substantially decreases the conservatism in the result. The numerical implementation is a sequence of a global optimisation and an interval arithmetical step. A numerical example illustrates that the method can be applied to study influences of different kinds of uncertainties on the FRF result.

\section{REFERENCES}

[1] Elishakoff, I., "Essay on uncertainties in elastic and viscoelastic structures: from A.M. Freudenthal's criticisms to modern convex modelling," Computers \& Structures, Vol. 56, No. 6, 1995, pp. 871-895.

[2] Rao, S. and Sawyer, P., "Fuzzy Finite Element Approach for the Analysis of Imprecisely Defined Systems," AIAA Journal, Vol. 33, No. 12, 1995, pp. 2364-2370.

[3] Moens, D. and Vandepitte, D., "Fuzzy Finite Element Method for Frequency Response Function Analysis of Uncertain Structures," AIAA Journal, Vol. 40, No. 1, 2002, pp. 126-136.
[4] Zadeh, L., "Fuzzy sets," Information and Control, Vol. 8, 1965, pp. 338-353.

[5] Chen, L. and Rao, S., "Fuzzy Finite-Element Approach for the Vibration Analysis of ImpreciselyDefined Systems," Finite Elements in Analysis and Design, Vol. 27, 1997, pp. 69-83.

[6] Moens, D., A Non-Probabilistic Finite Element Approach for Structural Dynamic Analysis with Uncertain Parameters, PhD thesis, K.U.Leuven, Leuven, 2002.

[7] Hitchings, D., A Finite Element Dynamics Primer, chap. A Finite Element Dynamics Primer, NAFEMS, Glasgow, 1992, pp. 170-196. 

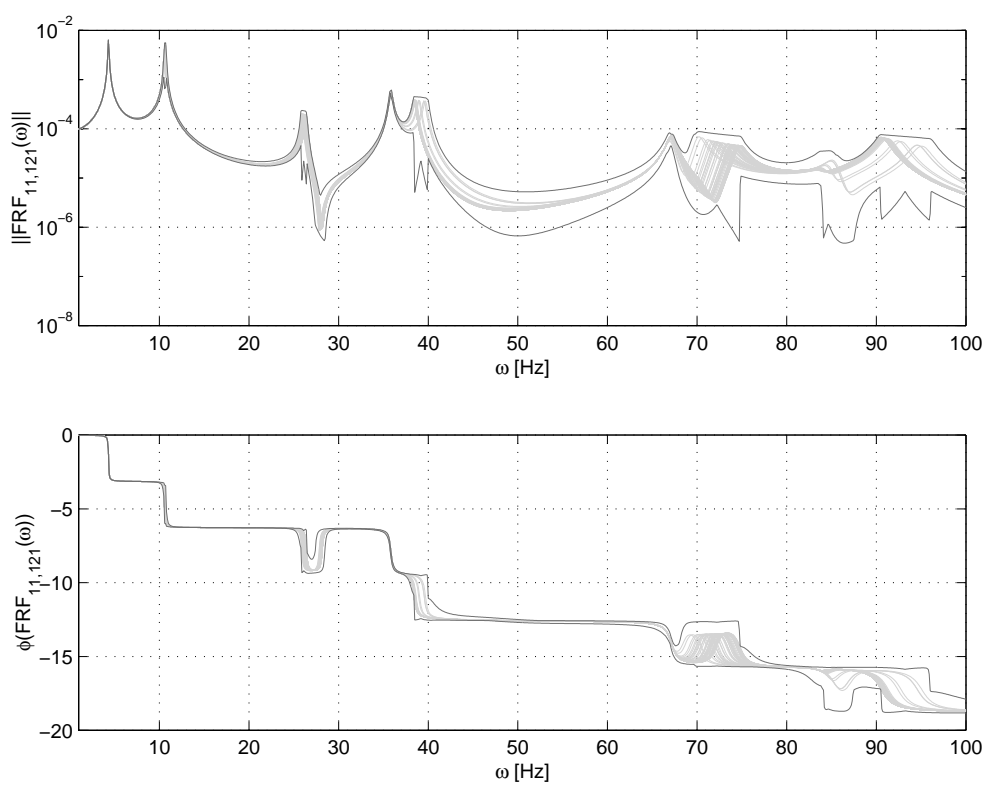

Figure 4: Envelope FRF for clamped plate with uncertain vertical boundary stiffness (MC with uniform distribution)

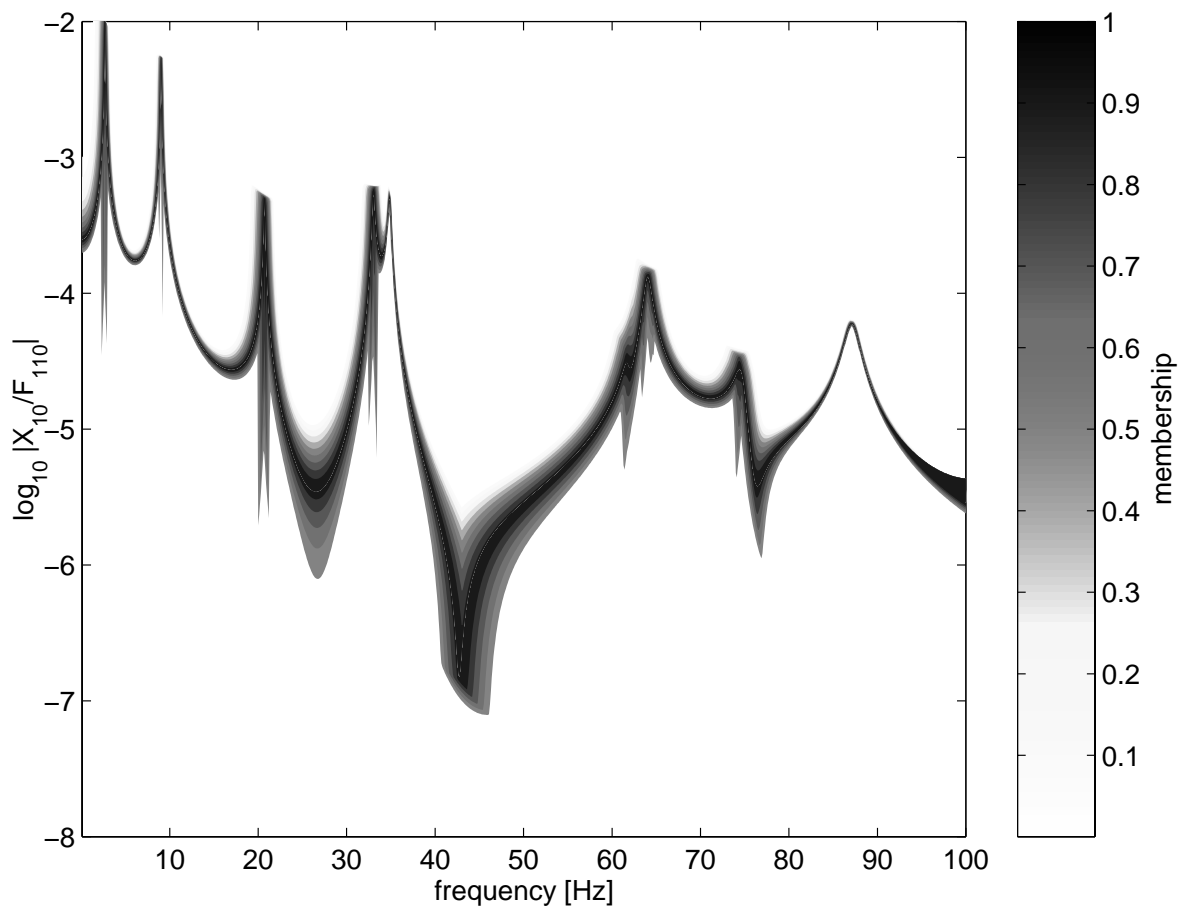

Figure 5: Fuzzy FRF between the free corners of the plate model 